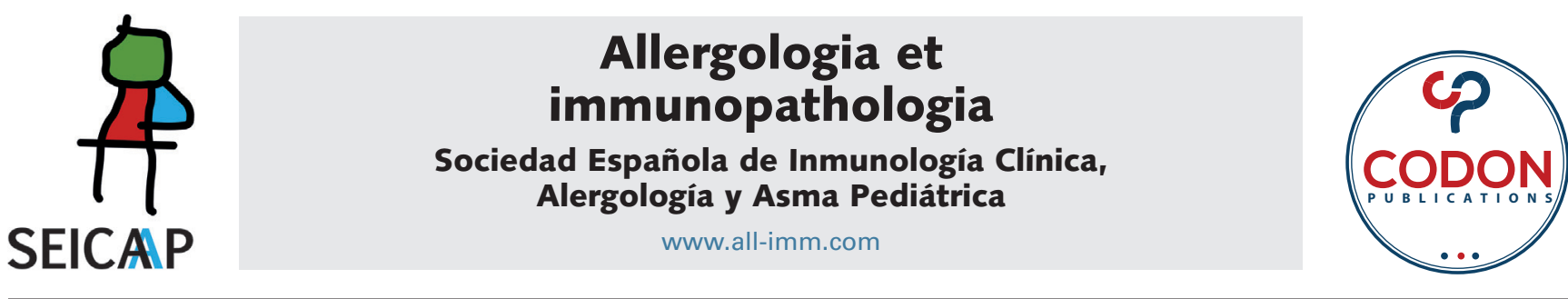

\title{
Real life study of the use of omalizumab for pediatric patients with multiple food allergies
}

\author{
Judit Barrena Crespo ${ }^{\mathrm{a} \dagger}$, Marta Viñas Domingo ${ }^{\mathrm{b}}$, Nora Hernández Arauzo \\ M. José Castillo ${ }^{\mathrm{b} \dagger}$, M. Belén Delavalle ${ }^{\mathrm{b}}$, M. Pilar Saura Foix ${ }^{\mathrm{b}}$, \\ Davinia Vázquez Sánchez ${ }^{\mathrm{a} \dagger \mathrm{t}}$, Adriana Izquierdo-Domínguez ${ }^{\mathrm{b*}}$
}

\begin{abstract}
${ }^{a}$ Department of Pediatric, Consorci Sanitari de Terrassa, Barcelona, Spain
${ }^{b}$ Department of Allergology, Consorci Sanitari de Terrassa, Barcelona, Spain

†Judit Barrena Crespo, Marta Viñas Domingo, Nora Hernández Arauzo: designed and prepared the manuscripts and collect data and final approval of the manuscript.

${ }^{\text {"M}}$. José Castillo, Belén Delavalle, Pilar Saura Foix: collected data and analyzed results and final approval of the manuscript. "Davinia Vázquez Sánchez, Adriana Izquierdo-Domínguez: prepared and reviewed the manuscript and final approval of the manuscript.
\end{abstract}

\author{
KEYWORDS \\ Multiple food allergy; \\ Oral Immunotherapy; \\ omalizumab, \\ anaphylaxis; \\ total IgE; \\ Food Allergy Quality \\ of Life Questionnaire \\ - Parent Form
}

\begin{abstract}
Background: Multiple food allergies (MFAs) affect $30 \%$ of the child population with food allergy. The current treatment is the exclusion diet, which frequently affects the quality of life for these patients. The objective of the study was to describe the effect of omalizumab treatment in children diagnosed with MFAs who experienced frequent anaphylactic reactions and the impact on their quality of life.

Material and methods: A descriptive observational study. Patients with severe food restrictions and high-risk due to multiple episodes of anaphylaxis were included. The allergy was confirmed by compatible clinical, skin tests, positive specific IgE and oral food challenges (OFCs). Omalizumab treatment was initiated and the impact on the life quality of patients and their families was assessed using the validated Food Allergy Quality of Life QuestionnaireParent Form.

Results: Five patients with an average age at diagnosis of 3.58 years (range between 1.5-7.9 years), were diagnosed with MFAs. All patients presented with anaphylaxis. All patients were treated with omalizumab between 2013 and 2019. Omalizumab treatment was initiated at a mean age of 6.05 years (range between 4.5-8.25 years). All patients have undergone OFC to reintroduce food successfully. 2 patients had their dose of omalizumab reduced by half, and 1 patient has had the time interval extended between administrations due to the maintenance of food tolerance. No immediate local or systemic adverse reactions were documented. Two patients have commenced omalizumab administration at home without incident.

Conclusions: Children with MFAs who are treated with omalizumab do not show reactions in response to most of the foods to which they previously had anaphylaxis. Consequently, these
\end{abstract}

*Corresponding author: Adriana Izquierdo-Dominguez. c. Vilana 12, 194 Office, 08022 Barcelona, Spain. Email address:

adrianaeizquierdo@hotmail.com 
patients were able to significantly expand the variety of their diet, improving the life quality and avoid anaphylaxis following the inadvertent intake of these foods.

(c) 2021 Codon Publications. Published by Codon Publications.

\section{Introduction}

Food allergies affect approximately $8 \%$ of children in the world, ${ }^{1-5}$ with the prevalence increasing in recent years. ${ }^{6}$ Moreover, up to $40 \%$ of children with food allergies can experience severe adverse reactions, ${ }^{1,4}$ which occur more frequently in teenagers (14-17 years) and young people with multiple food allergies (MFAs). ${ }^{1,7}$ Approximately 30\% of children with food allergies have MFAs, ${ }^{1}$ which lead to restrictive diets, impaired quality of life $(\mathrm{Q} o \mathrm{~L})$, greater economic expense, and a constant risk of accidental and severe allergic reactions.

Since there is currently no approved specific treatment in clinical practice for such cases, a restrictive diet devoid of the food allergen traditionally represents the only available therapy. Several studies have considered oral immunotherapy (OIT) as a therapeutic option for the treatment of food allergies, including allergies to milk and eggs. ${ }^{8}$ However, since OIT is often an unviable treatment for individuals with MFAs, it remains poorly studied in this patient population. ${ }^{9}$ It has been suggested that anti-immunoglobulin $E$ (e.g., omalizumab) may represent an alternative treatment strategy to prevent or reduce allergic reactions in pediatric patients with MFAs. ${ }^{9}$ Pilot studies have reported preliminary results of omalizumab treatment, describing a reduction in anaphylaxis following accidental food intake in patients with food allergies, but it remains inadequately studied. ${ }^{9}$

The objective of our study was to describe the effect of omalizumab treatment in five children diagnosed with MFAs who experienced frequent episodes of anaphylaxis and could not receive OIT for multiple foods.

\section{Methods}

\section{Study design}

A descriptive observational study was conducted to investigate the effect of omalizumab treatment in five children with anaphylactic reactions and diagnosed with MFAs and severe restrictive diets. The pediatric patients were recruited from the Allergy Service. The diagnosis of MFAs was made using the following compatible clinical tests: (1) in vitro: specific IgE (ImmunoCAP Specific IgE Thermophisher Uppsala Sweden) and molecular allergy profile with the ImmunoCAP ISAC ${ }^{\circledR}$ test (Immuno Solid-Phase Allergen Chip); and (2) in vivo: skin test and oral food challenge (OFC) in which the presence of allergic response or tolerance could be confirmed.

\section{Omalizumab treatment and oral food challenge}

The doses of omalizumab in the patients were adjusted to the weight and the initial total IgE values (according to the data sheet). Since Patient 3 weighed less than $20 \mathrm{~kg}$, the dose was adjusted every 4 weeks. Omalizumab was prescribed as a medication for compassionate use, and written informed consent was signed by the parents or legal guardians. The possibility of administering omalizumab at home was also recently evaluated.

After 6 months of omalizumab treatment, we started with OFC, first choosing foods with which the patient had presented with mild allergic reactions, and subsequently testing foods to which the patient had exhibited more severe allergic reactions. The OFCs were performed following the doses recommended by the PRACTALL consensus report, ${ }^{10}$ with a minimum interval of 1 week between OFC. Both the skin and in vitro tests were repeated after 2 years of omalizumab treatment.

\section{Measurement of quality of life}

A validated food allergy QoL questionnaire (FAQLQ-PF) was obtained prior to the start of the treatment with omalizumab and also immediately after starting the introduction of some foods (after 2 years of omalizumab treatment).

\section{Results}

Five patients with an average age at diagnosis of 3.58 years (range: 1.5-7.9 years), were diagnosed with MFAs. All patients presented with anaphylaxis. All patients were treated with omalizumab between 2013 and 2019. Omalizumab treatment was initiated at a mean age of 6.05 years (range: 4.5-8.25 years). Demographic characteristics and comorbidities are described in Table 1. Table 2 lists the foods to which the patients had a reaction or where sensitization was detected and detailed characteristics of the five patients with MFAs pre- and during omalizumab treatment. In most cases, the diagnosis was made using compatible symptoms, positive skin tests, and specific lgE. The OFCs were only performed on foods not previously ingested with low sensitization, and confirmed the allergy in all cases.

After 6 months of omalizumab treatment, the patients underwent an OFC to the different allergic foods. The OFC was successful in all but two patients, patient number 4 presented with urticaria in response to cooked egg white, and it was decided that they should start treatment with OIT for raw egg white, which was associated with a favorable response. Patient number 3 presented with urticaria following the ingestion of hazelnuts.

We performed a skin test after several involved foods had been introduced, and again after 2 years of omalizumab treatment, which showed a decrease in the papule size compared to baseline papule. Blood tests were performed in four patients, and revealed that the total IgE 


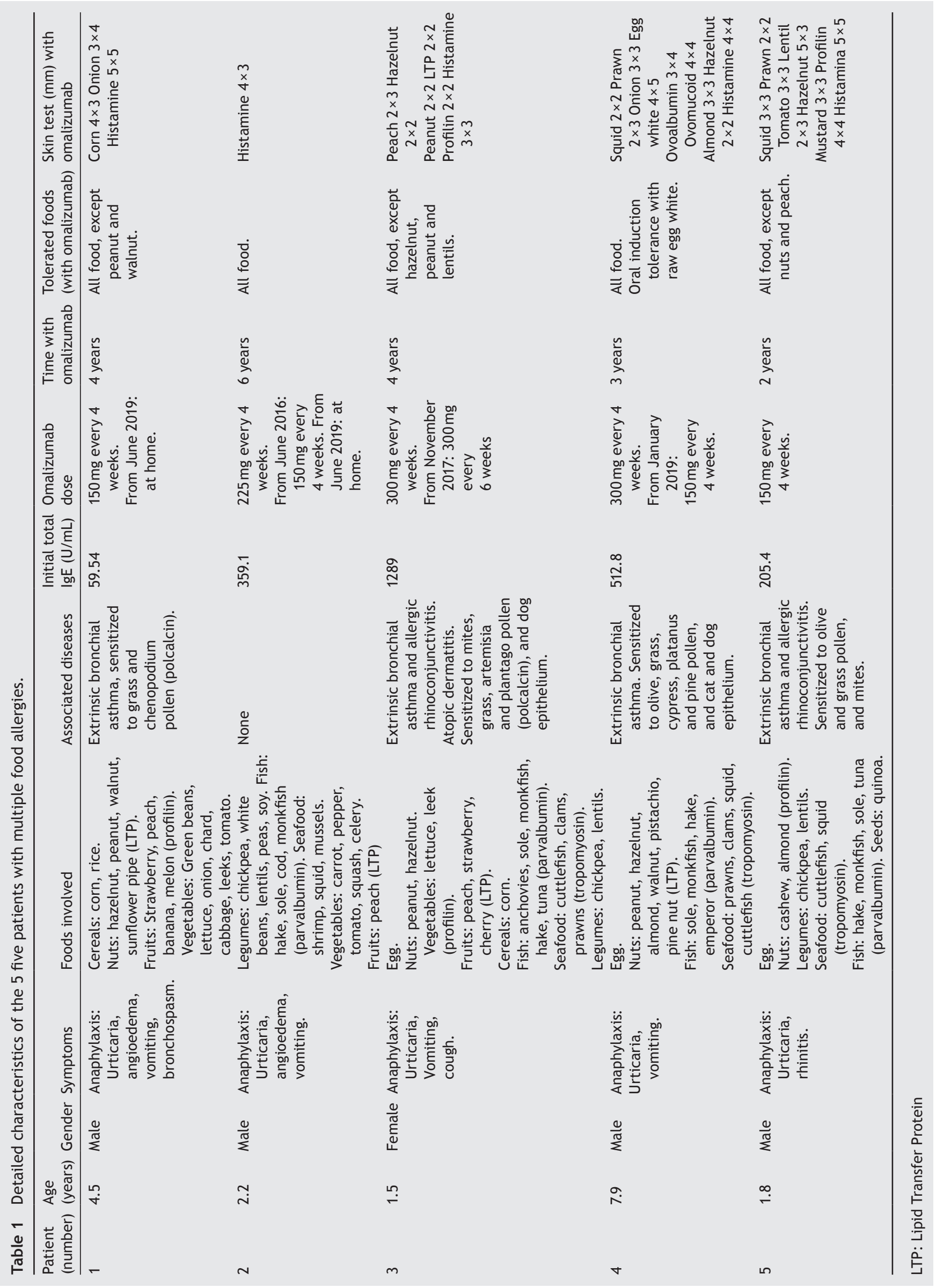


Table 2 Detailed characteristics of the five patients with multiple food allergy pre- and during omalizumab treatment.

\begin{tabular}{|c|c|c|c|c|c|c|c|c|c|c|c|c|c|}
\hline $\begin{array}{l}\text { Patient } \\
\text { (number) }\end{array}$ & $\begin{array}{l}\text { Foods that } \\
\text { are causing } \\
\text { reactions } \\
\text { (pre-OMZ) }\end{array}$ & $\begin{array}{l}\text { Prick } \\
\text { test }(\mathrm{mm}) \\
\text { pre-OMZ }\end{array}$ & $\begin{array}{l}\text { slgE } \\
\text { (kU/L) } \\
\text { pre-OMZ }\end{array}$ & $\begin{array}{l}\text { OFC } \\
\text { pre- } \\
\text { OMZ }\end{array}$ & $\begin{array}{l}\text { Dose } \\
\text { toler- } \\
\text { ability } \\
\text { (gr) }\end{array}$ & U/A & $\mathrm{Gl}$ & $\mathrm{ms}$ & $\begin{array}{l}\text { Prick test } \\
(\mathrm{mm}) \\
\text { during } \\
\mathrm{OMZ}\end{array}$ & $\begin{array}{l}\text { slgE } \\
\text { (kU/L) } \\
\text { during } \\
\text { OMZ }\end{array}$ & $\begin{array}{l}\text { ISAC } \\
\text { test } \\
\text { (ISU-E) }\end{array}$ & $\begin{array}{l}\text { OFC } \\
\text { during } \\
\text { OMZ }\end{array}$ & $\begin{array}{l}\text { Dose } \\
\text { toler- } \\
\text { ability } \\
\text { (gr) }\end{array}$ \\
\hline \multirow[t]{18}{*}{1} & Corn & $6 \times 8$ & 19,2 & No & & + & & + & $4 \times 3$ & 6.01 & & - & 30 \\
\hline & Rice & $5 \times 5$ & 5.72 & No & & & + & & - & 1.97 & & - & 30 \\
\hline & Hazelnut & $3 \times 3$ & 2.37 & No & & + & & & - & 0.59 & & - & 15 \\
\hline & Peanut & $5 \times 4$ & 6.27 & No & & + & & + & - & 5 & & No & Avoid \\
\hline & Walnut & $3 \times 4$ & 14 & No & & + & & + & - & 4.27 & & No & Avoid \\
\hline & Sunflower pipe & $3 \times 3$ & 1.07 & No & & + & & & - & $<0.10$ & & - & 20 \\
\hline & Strawberry & $3 \times 4$ & 19.7 & No & & & + & & - & 8.2 & & - & 50 \\
\hline & Peach & $5 \times 5$ & 17.6 & No & & & & & - & 5.82 & & - & 100 \\
\hline & Banana & $2 \times 2$ & 0.98 & + & 10 & + & & & - & $<0.10$ & & - & 100 \\
\hline & Melon & $3 \times 3$ & 2.35 & No & & & + & & - & 0.88 & & No & $\mathrm{T}$ \\
\hline & Green beans & $3 \times 3$ & 4.43 & No & & & & & - & 2.03 & & - & 100 \\
\hline & Lettuce & $3 \times 4$ & 1.37 & + & 5 & & + & & - & 0.55 & & - & 30 \\
\hline & Onion & $6 \times 4$ & 62.2 & No & & & & & $3 \times 4$ & 20.7 & & - & 30 \\
\hline & Chard & $5 \times 7$ & & No & & & + & + & - & & & - & 100 \\
\hline & Cabbage & $8 \times 6$ & 2.39 & No & & & + & + & - & 2.63 & & - & $\mathrm{T}$ \\
\hline & Leek & $9 \times 8$ & & No & & & + & + & - & & & - & 30 \\
\hline & Potato & $2 \times 2$ & 0.76 & + & 10 & & + & & - & $<0.10$ & & - & 100 \\
\hline & \multicolumn{2}{|c|}{ Total IgE (U/mL) } & 218.9 & & & & & & & 184 & & - & \\
\hline \multirow[t]{24}{*}{2} & Chickpea & $10 \times 5$ & 26.4 & No & & + & + & & - & 18.8 & & - & 60 \\
\hline & White bean & $5 \times 5$ & 5.67 & No & & + & + & & - & & & - & 60 \\
\hline & Pea & $9 \times 5$ & 23.1 & No & & & & & - & 15.4 & & - & 60 \\
\hline & Lentil & $5 \times 6$ & 20.9 & No & & + & + & + & - & 18 & & - & 60 \\
\hline & Soy & $3 \times 3$ & 2.75 & No & & + & & & - & & $\begin{array}{r}\text { rGly m } \\
\text { 4: } 14 \\
\text { nGly m } \\
\text { 5: } 1.1 \\
\text { nGly m } \\
\text { 6: } 0.7\end{array}$ & - & 30 \\
\hline & Hake & $5 \times 5$ & 6.86 & No & & + & + & & - & 0.98 & & - & 60 \\
\hline & Sole & $7 \times 5$ & 3 & No & & + & + & & - & 0.35 & & - & 60 \\
\hline & Tuna & $3 \times 4$ & 1.17 & + & & + & & & - & $<0.10$ & & - & 60 \\
\hline & Cod & $7 \times 5$ & 7.02 & No & & + & + & & - & & $\begin{array}{c}\text { rGad c } 1 \\
0.4\end{array}$ & :No & $\mathrm{T}$ \\
\hline & Monkfish & $5 \times 6$ & & No & & + & + & & - & & & No & $\mathrm{T}$ \\
\hline & Shrimp & $3 \times 3$ & 0.84 & No & & & & & - & & $\begin{array}{r}\text { nPen } \mathrm{m} \\
\text { 1: } 0.7\end{array}$ & - & $\mathrm{T}$ \\
\hline & Squid & $3 \times 3$ & 0.26 & + & 6 & + & & + & - & 0.42 & & - & 30 \\
\hline & Mussels & $2 \times 2$ & 1.41 & No & & + & & + & - & & & - & $\mathrm{T}$ \\
\hline & Carrot & $7 \times 5$ & 11 & No & & + & + & & - & 13 & & - & 30 \\
\hline & Pepper & $3 \times 3$ & 1.65 & + & 3 & + & + & & $2 \times 3$ & & & - & 30 \\
\hline & Cauliflower & $5 \times 4$ & 2.07 & No & & & & & - & 7.12 & & - & $\mathrm{T}$ \\
\hline & Squash & $4 \times 4$ & 4.8 & No & & & & & - & & & No & $\mathrm{T}$ \\
\hline & Chard & $5 \times 5$ & & No & & + & & & - & & & No & $\mathrm{T}$ \\
\hline & Onion & $4 \times 3$ & 3.48 & No & & + & + & & - & 9.61 & & No & $\mathrm{T}$ \\
\hline & Green beans & $4 \times 4$ & 6.47 & No & & + & & & - & 21.2 & & - & 100 \\
\hline & Lettuce & $3 \times 3$ & 0.74 & + & 10 & & + & & - & 1.33 & & No & 30 \\
\hline & Tomato & $4 \times 4$ & 2.14 & No & & + & & & - & 7.38 & & - & 30 \\
\hline & Spinach & $3 \times 3$ & 3.01 & No & & & & & - & 6.24 & & No & $\mathrm{T}$ \\
\hline & Celery & $3 \times 3$ & 3.03 & No & & & & & - & 6.68 & $\begin{array}{c}\text { rApi g 1: } \\
2.8\end{array}$ & No & $\mathrm{T}$ \\
\hline
\end{tabular}


Table 2 (Continued)

\begin{tabular}{|c|c|c|c|c|c|c|c|c|c|c|c|c|c|}
\hline $\begin{array}{l}\text { Patient } \\
\text { (number) }\end{array}$ & $\begin{array}{l}\text { Foods that } \\
\text { are causing } \\
\text { reactions } \\
\text { (pre-OMZ) }\end{array}$ & $\begin{array}{l}\text { Prick } \\
\text { test }(\mathrm{mm}) \\
\text { pre-OMZ }\end{array}$ & $\begin{array}{l}\text { slgE } \\
(\mathrm{kU} / \mathrm{L}) \\
\text { pre-OMZ }\end{array}$ & $\begin{array}{l}\text { OFC } \\
\text { pre- } \\
\text { OMZ }\end{array}$ & $\begin{array}{l}\text { Dose } \\
\text { toler- } \\
\text { ability } \\
\text { (gr) }\end{array}$ & U/A & GI & $\mathrm{ms}$ & $\begin{array}{l}\text { Prick test } \\
(\mathrm{mm}) \\
\text { during } \\
\text { OMZ }\end{array}$ & $\begin{array}{l}\text { slgE } \\
\text { (kU/L) } \\
\text { during } \\
\text { OMZ }\end{array}$ & $\begin{array}{l}\text { ISAC } \\
\text { test } \\
\text { (ISU-E) }\end{array}$ & $\begin{array}{l}\text { OFC } \\
\text { during } \\
\text { OMZ }\end{array}$ & $\begin{array}{l}\text { Dose } \\
\text { toler- } \\
\text { ability } \\
\text { (gr) }\end{array}$ \\
\hline & Hazelnut & $5 \times 4$ & 9.08 & No & & + & & & - & 0.88 & $\begin{array}{c}\text { rCor a 8: } \\
0.4\end{array}$ & & 20 \\
\hline & Peach & $6 \times 6$ & 14.5 & No & & + & & + & - & & $\begin{array}{c}\text { rPru p 3: } \\
0.8\end{array}$ & & 100 \\
\hline & Total IgE (U/mL) & & 359.1 & & & & & & & 487 & & & \\
\hline \multirow[t]{19}{*}{3} & Egg & $\begin{array}{c}\text { EW: } 3 \times 4 \\
\text { OVA } 4 \times 3 \\
\text { OVM: } \\
3 \times 3\end{array}$ & $\begin{array}{l}\text { EW: } 2.47 \\
\text { OVA } 2.5 \\
\text { OVM: } 1.11\end{array}$ & + & 4 (EW) & + & + & + & - & $\begin{array}{c}\text { EW: } \\
1.45 ; \\
\text { OVA } \\
1.28 \\
\text { OVM: } \\
0.48\end{array}$ & & - & $\begin{array}{l}3.3 \\
\quad(\text { REW })\end{array}$ \\
\hline & Peanut & $10 \times 9$ & 50.5 & No & & + & + & + & $2 \times 2$ & & $\begin{array}{r}\text { rAra h } \\
9: 1\end{array}$ & No & Avoid \\
\hline & Walnut & $9 \times 9$ & 51.7 & No & & + & + & & - & & $\begin{array}{c}\text { rJug r 1: } \\
3.2 \\
\text { nJug r 2: } \\
0.7 \\
\text { nJug r } 3: \\
1.6\end{array}$ & $\begin{array}{l}- \\
: \\
:\end{array}$ & 30 \\
\hline & Almond & $7 \times 6$ & 28.1 & No & & & & & - & 2.45 & & - & 30 \\
\hline & Hazelnut & $7 \times 8$ & 49.2 & No & & + & & & $2 \times 2$ & & $\begin{array}{c}\text { rCor a 8: } \\
2.6\end{array}$ & & 10 \\
\hline & Lettuce & $4 \times 5$ & 8.84 & No & & + & & & - & 1.03 & & - & 30 \\
\hline & Green beans & $5 \times 5$ & 20.1 & No & & + & + & & - & 3.67 & & - & 30 \\
\hline & Lentil & $5 \times 5$ & 15 & No & & + & & & - & 11 & & No & Avoid \\
\hline & Chickpea & $4 \times 5$ & 9.32 & No & & + & & & - & 2.98 & & - & 30 \\
\hline & Peach & $10 \times 9$ & 74.7 & No & & + & + & & $2 \times 3$ & & $\begin{array}{c}\text { rPru p 3: } \\
1.4\end{array}$ & No & Avoid \\
\hline & Strawberry & $7 \times 6$ & 30.9 & No & & + & & & - & 4.2 & & - & 30 \\
\hline & Cherry & $6 \times 6$ & 24.7 & No & & + & + & & - & 0.34 & & No & $\mathrm{T}$ \\
\hline & Corn & $5 \times 5$ & 14.8 & No & & + & & & - & 11.5 & & - & 60 \\
\hline & Sole & $3 \times 3$ & 2.8 & - & 60 & & & & - & & & No & $\mathrm{T}$ \\
\hline & Cod & $3 \times 4$ & 7.89 & No & & & + & & - & 0.17 & & No & $\mathrm{T}$ \\
\hline & Hake & $3 \times 4$ & 5.5 & + & 10 & & + & & - & 0.21 & & - & 60 \\
\hline & Shrimp & $3 \times 3$ & 3.89 & + & 10 & + & & & - & 3.71 & & - & 30 \\
\hline & Squid & $3 \times 3$ & 4.51 & No & & & & & - & & $\begin{array}{r}\text { nPen m } \\
\text { 1: } 2.9\end{array}$ & - & 30 \\
\hline & Total IgE $(\mathrm{U} / \mathrm{mL})$ & & 1289 & & & & & & & 892.7 & & & \\
\hline \multirow[t]{8}{*}{4} & Egg & $\begin{array}{l}\text { EW: } 7 \times 8 \\
\text { OVA } 6 \times 7 \\
\text { OVM: } \\
7 \times 8\end{array}$ & $\begin{array}{l}\text { EW: } 22.8 \\
\text { OVA } 8.68 \\
\text { OVM: } \\
14.5\end{array}$ & No & & + & + & + & $\begin{array}{l}\text { EW } 4 \times 5 \\
\text { OVA } 3 \times 4 \\
\text { OVM } 4 \times 4\end{array}$ & $\begin{array}{l}\text { EW: } 6.8 \\
\text { OVA } 4.76 \\
\text { OVM: } \\
5.57\end{array}$ & & + & 3 (CEW) \\
\hline & Peanut & $3 \times 4$ & 1.14 & No & & & & & - & 0.34 & & - & 20 \\
\hline & Hazelnut & $3 \times 3$ & 0.98 & No & & + & + & & $2 \times 2$ & 0.37 & & - & 20 \\
\hline & Cashew & $4 \times 4$ & 2.52 & No & & + & + & & - & 1.01 & & - & 20 \\
\hline & Almond & $3 \times 3$ & 0.58 & No & & & & & $3 \times 3$ & 0.55 & & - & 20 \\
\hline & Walnut & $3 \times 2$ & 0.29 & + & 5 & + & & & - & $<0.10$ & & - & 20 \\
\hline & Pistachio & $4 \times 4$ & 3.59 & No & & & & & - & & & No & $T$ \\
\hline & Pine nut & $3 \times 2$ & 0.39 & No & & & & & - & & & No & $\mathrm{T}$ \\
\hline
\end{tabular}


Table 2 (Continued)

\begin{tabular}{|c|c|c|c|c|c|c|c|c|c|c|c|c|c|}
\hline $\begin{array}{l}\text { Patient } \\
\text { (number) }\end{array}$ & $\begin{array}{l}\text { Foods that } \\
\text { are causing } \\
\text { reactions } \\
\text { (pre-OMZ) }\end{array}$ & $\begin{array}{l}\text { Prick } \\
\text { test }(\mathrm{mm}) \\
\text { pre-OMZ }\end{array}$ & $\begin{array}{l}\text { slgE } \\
\text { (kU/L) } \\
\text { pre-OMZ }\end{array}$ & $\begin{array}{l}\text { OFC } \\
\text { pre- } \\
\text { OMZ }\end{array}$ & $\begin{array}{l}\text { Dose } \\
\text { toler- } \\
\text { ability } \\
\text { (gr) }\end{array}$ & U/A & GI & ms & $\begin{array}{l}\text { Prick test } \\
(\mathrm{mm}) \\
\text { during } \\
\text { OMZ }\end{array}$ & $\begin{array}{l}\text { slgE } \\
\text { (kU/L) } \\
\text { during } \\
\text { OMZ }\end{array}$ & $\begin{array}{l}\text { ISAC } \\
\text { test } \\
\text { (ISU-E) }\end{array}$ & $\begin{array}{l}\text { OFC } \\
\text { during } \\
\text { OMZ }\end{array}$ & $\begin{array}{l}\text { Dose } \\
\text { toler- } \\
\text { ability } \\
\text { (gr) }\end{array}$ \\
\hline & Tuna & $3 \times 3$ & 1.05 & + & 15 & & + & & - & $<0.10$ & & - & 60 \\
\hline & Hake & $3 \times 4$ & 1.43 & No & & & + & & - & $<0.10$ & & No & $\mathrm{T}$ \\
\hline & Cod & $4 \times 4$ & 1.7 & No & & & + & & - & 0.12 & & No & $\mathrm{T}$ \\
\hline & Shrimp & $3 \times 3$ & 1.53 & No & & + & & + & $2 \times 3$ & 0.85 & & - & 30 \\
\hline & Squid & $2 \times 2$ & 0.67 & No & & + & & & $2 \times 2$ & 0.37 & & - & 60 \\
\hline & Clams & $2 \times 2$ & 0.43 & No & & + & & & - & & & No & $\mathrm{T}$ \\
\hline & \multicolumn{2}{|c|}{ Total lgE (U/mL) } & \multicolumn{2}{|c|}{523.3} & & & & & - & 247.9 & & & \\
\hline \multirow[t]{18}{*}{5} & Egg & $\begin{array}{l}\text { EW: } 3 \times 3 \text {; } \\
\text { OVA } 3 \times 4 \text {, } \\
\text { OVM: } \\
2 \times 2\end{array}$ & \multicolumn{2}{|c|}{$\begin{array}{l}\text { EW: } 2.65 ;+ \\
\text { OVA } 2.08 \\
\text { OVM: } 0.5\end{array}$} & \multicolumn{4}{|c|}{5 (REW) + } & - & & & - & $\begin{array}{l}3.3 \\
\quad \text { (REW) }\end{array}$ \\
\hline & Cashew & $3 \times 3$ & 4.36 & No & & + & + & & - & & & No & Avoid \\
\hline & Pistachio & $3 \times 3$ & 4.76 & No & & & & & - & & & No & Avoid \\
\hline & Hazelnut & $6 \times 5$ & 3.5 & No & & & & & $5 \times 3$ & & & No & Avoid \\
\hline & Almond & $3 \times 3$ & 3.3 & + & 3 & + & + & & - & & & No & Avoid \\
\hline & Sunflower pipe & $2 \times 3$ & 3.16 & No & & & & & - & & & No & Avoid \\
\hline & Chickpea & $5 \times 4$ & 11 & No & & + & & + & - & & & - & 60 \\
\hline & Pea & $4 \times 4$ & 12.5 & No & & & & & - & & & - & 60 \\
\hline & White bean & $3 \times 2$ & 1.38 & + & 10 & & & & - & & & No & $\mathrm{T}$ \\
\hline & Lentil & $5 \times 6$ & 11.9 & No & & + & & + & $2 \times 3$ & & & - & 60 \\
\hline & Squid & $3 \times 3$ & 0.42 & No & & + & & + & $3 \times 3$ & & & - & 60 \\
\hline & Hake & $4 \times 5$ & 5.88 & No & & + & + & & - & & & - & 60 \\
\hline & Cod & $6 \times 5$ & 9.7 & No & & + & + & & - & & & - & 60 \\
\hline & Sole & $4 \times 4$ & 5.75 & No & & & + & & - & & & - & 60 \\
\hline & Tuna & $3 \times 3$ & 2.08 & No & & & + & & - & & & - & 60 \\
\hline & Peach & $3 \times 4$ & 1.09 & No & & + & & & - & & & No & Avoid \\
\hline & Quinoa & $2 \times 2$ & 0.93 & + & 30 & + & & + & - & & & - & 30 \\
\hline & Total IgE (U/mL) & & 205.4 & & & & & & & & & & \\
\hline
\end{tabular}

OMZ: omalizumab; EW: egg white; OVA: ovalbumin; OVM: ovomucoid; REW: raw egg white; CEW: cooked egg white; T: tolerated; U: urticaria; A: angioedema, GI: gastrointestinal; R: respiratory; ISAC: Immuno Solid-Phase Allergen Chip.

*(-): no reaction *(+): allergic reaction *No: unrealized.

Table 3 Characteristics and doses of omalizumab treatment in each patient.

\begin{tabular}{|c|c|c|c|c|c|c|c|c|}
\hline $\begin{array}{l}\text { Patient } \\
\text { (number) }\end{array}$ & $\begin{array}{c}\text { Age (years) } \\
\text { at OMZ } \\
\text { start }\end{array}$ & $\begin{array}{c}\text { Total IgE } \\
(\mathrm{U} / \mathrm{mL}) \\
\text { pre- OMZ }\end{array}$ & $\begin{array}{c}\text { Weight } \\
\text { (kg) }\end{array}$ & $\begin{array}{l}\text { OMZ initial } \\
\text { dose }\end{array}$ & $\begin{array}{l}\text { Dose change } \\
\text { time }\end{array}$ & $\begin{array}{l}\text { OMZ current } \\
\text { dose }\end{array}$ & $\begin{array}{l}\text { Treatment } \\
\text { at home }\end{array}$ & $\begin{array}{l}\text { Treatment time } \\
\text { (years) with } \\
\text { OMZ (currently) }\end{array}$ \\
\hline 1 & 8 & 218.9 & 26 & $\begin{array}{l}150 \text { mg every } \\
4 \text { weeks }\end{array}$ & - & $\begin{array}{l}150 \mathrm{mg} \text { every } \\
4 \text { weeks }\end{array}$ & $\begin{array}{l}\text { Yes: from } \\
\text { June } 2019\end{array}$ & 4.7 \\
\hline 2 & 4.9 & 359.1 & 20 & $\begin{array}{l}225 \text { mg every } \\
4 \text { weeks }\end{array}$ & June 2016 & $\begin{array}{l}150 \mathrm{mg} \text { every } \\
4 \text { weeks }\end{array}$ & $\begin{array}{l}\text { Yes: from } \\
\text { June } 2019\end{array}$ & 6.2 \\
\hline 3 & 4.6 & 1289 & 17 & $\begin{array}{l}300 \text { mg every } \\
4 \text { weeks }\end{array}$ & November 2017 & $\begin{array}{l}300 \mathrm{mg} \text { every } \\
6 \text { weeks }\end{array}$ & No & 4.7 \\
\hline 4 & 8.3 & 512.8 & 28 & $\begin{array}{l}300 \mathrm{mg} \text { every } \\
4 \text { weeks }\end{array}$ & January 2019 & $\begin{array}{l}150 \mathrm{mg} \text { every } \\
4 \text { weeks }\end{array}$ & No & 3.9 \\
\hline 5 & 4.5 & 205.4 & 20 & $\begin{array}{l}150 \mathrm{mg} \text { every } \\
4 \text { weeks }\end{array}$ & - & $\begin{array}{l}150 \mathrm{mg} \text { every } \\
4 \text { weeks }\end{array}$ & No & 2.3 \\
\hline
\end{tabular}

OMZ: omalizumab. 
values decreased in three patients during treatment with omalizumab, but increased in one patient. Similar results were also found regarding the specific IgE values, which decreased or increased regardless of tolerance.

At present, the patients have been treated with omalizumab for 2.3-6.2 years. Table 3 describes the omalizumab treatment received by each patient, dosage, and weaning. Two patients had their dose of omalizumab reduced by half, and one patient had the time interval extended between administrations due to the maintenance of food tolerance. No immediate local or systemic adverse reactions were documented, nor have any delayed reactions been reported. After rigorous training to the family on the use of omalizumab and obtaining prior informed consent, two patients have commenced omalizumab administration at home without incident.

The results of the food allergy QoL questionnaire indicate that the parents of the patients generally perceived a significant improvement in the health status of their children. Parents also reported reduced stress associated with the allergy in both the patient and in the families, as well as a significant decrease in the limitations of the patients' activities in their daily life.

\section{Discussion}

The findings of our study indicate that pediatric patients with MFAs who are treated with omalizumab do not show reactions in response to most of the foods to which they previously had severe allergic reactions. Consequently, these patients were able to significantly extend the variety of their diet and avoid several allergic reactions following the inadvertent intake of these foods.

This series involved five pediatric patients with severe reactions to multiple foods and for whom treatment with ITO to multiple foods was challenging. Some recent studies have demonstrated the efficacy of OIT combined with omalizumab. The study by Sampson et al. ${ }^{11}$ provides data showing that treatment with peanut OIT combined with omalizumab for 24 weeks can increase peanut tolerance compared to placebo-treated subjects $(p=0.054)$. Another study $^{12}$ used a combination of cow's milk OIT with omalizumab over 10 months, and found that $88.9 \%$ of the subjects could tolerate $10 \mathrm{gr}$ of cow's milk protein compared to the $71.4 \%$ of patients treated with the placebo. When omalizumab treatment was stopped and another OFC was performed 4 months later, sustained reactivity was achieved in $48.1 \%$ of the omalizumab group facing to $35.7 \%$ of the placebo group. ${ }^{12}$ Therefore, it was concluded that omalizumab improves the safety of OIT but has no effect on efficacy.

Bégin et al. ${ }^{13}$ investigated a combination OIT treatment for MFAs, which proposes the use of omalizumab pre-treatment for 16 weeks as an adjuvant therapy to perform desensitization treatment for MFAs. A total of $96 \%$ of reactions were of moderate intensity, with only one patient experiencing anaphylaxis; however, these reactions were observed to progressively decrease in frequency as the length of OIT increased..$^{13}$ In our experience, all patients had an increased tolerance threshold following omalizumab, even gaining the ability to tolerate foods that had previously produced severe allergic reactions. It is proposed that OFC be performed starting at 6 months of omalizumab treatment while continuing to deliver the treatment. Mild reactions (urticaria) were observed in only two patients, and the others tolerated the food. Patients with a negative OFC are recommended to continue eating the food frequently, but without fixed guidelines for periodicity or dosage of the food. At present, none of the patients have lost the achieved tolerance threshold.

We have tried to reduce the dose of omalizumab, aiming to stop administrating the drug in the future and check the sustained tolerance. To achieve this, we waited until the patients had received more than 3 years of treatment without presenting severe reactions. Moreover, the decrease in dose or extension of the interval between administrations has been successful. Consequently, we believe that safety can be increased if omalizumab is maintained in these high-risk patients.

Labrosse et al. ${ }^{14}$ reviewed the main studies in which omalizumab has been tested in OIT with food, observing greater safety and efficiency in the protocols; however, the long-term effects remain unknown. There is insufficient scientific evidence regarding how long omalizumab should be administered or how to perform weaning. Our experience suggests that maintaining omalizumab treatment for at least 3 years has been beneficial for our patients, and progressive weaning can help achieve the level of sustained tolerance.

The cost of omalizumab is another important factor to consider. Therefore, it is recommended that patients should be highly selective, with proven MFA, anaphylactic reactions, as well as restrictive diets and frequent reactions that alter their QoL. It must also be considered that patients with MFAs bear a significant psychological and economic burden, both for the healthcare system in generating multiple emergency visits and assistance from specialists, as well as families who must accommodate expensive and difficult diets. In our case series, we found an improvement in the QoL of patients and their families because they were able to extend their diet and did not experience any adverse reactions.

Fiocchi et al. ${ }^{15}$ studied patients with severe asthma treated with omalizumab, in which a small subgroup with MFAs was evaluated. The authors observed an increase in the tolerance threshold after 4-6 months of omalizumab treatment. ${ }^{15}$ An improvement in QoL was also observed, which was attributed to better asthma control and reduction in dietary restrictions.

Similar to the findings in our study, Mendonca et al. ${ }^{16}$ reported on three pediatric patients with MFAs and severe reactions who were treated with omalizumab, and observed a decreased frequency of reactions and an increase in QoL after 6 months to 1 year of treatment.

One of the limitations of our study is the sample size; however, this is a common limitation in other similar publications since omalizumab is currently rarely used to treat indication in food allergies. Although changes in the IgG4 values could not be analyzed in this study, we observed that the papule size was significantly decreased in each of the patients after 2 years of omalizumab treatment with maintained tolerance to allergenic food. In contrast, neither the total IgE nor specific IgE values followed a consistent pattern. In some cases, the values increased 
during treatment, as described in other studies that have used omalizumab for the treatment of severe asthma. ${ }^{17}$ In contrast, Sampson et al. ${ }^{11}$ showed that total IgE values decreased after treatment with omalizumab. In our experience, these values have not been a reliable parameter to predict the tolerability to food allergens.

In conclusion, treatment with omalizumab in children with MFAs who have presented with frequent severe allergic reactions and follow restrictive diets may represent an alternative safe treatment in a highly selective group of patients. Although it still has no indication for food allergy, parents perceive a significant improvement in the health status of their children. Besides, omalizumab reduces the stress associated with the allergy in both the patients and their families, as well as a significant decrease in the limitations of the patients' daily activities. Finally, with rigorous training to the families on the use of omalizumab and obtaining prior informed consent, selected patients could do home treatment.

\section{Conflicts of Interest}

The authors declare that they have no conflicts of interest.

\section{References}

1. Gupta RS, Springton EE, Warrier MR, Smith B, Kumar R, Pongracic J, et al. The prevalence, severity, and distribution of childhood food allergy in the United States. Pediatrics. 2011;128:9-17. https://doi.org/10.1542/peds.2011-0204

2. McGowan EC, Keet CA. Prevalence of self-reported food allergy in the National Health and Nutrition Examination Survey (NHANES) 2007-2010. J Allergy Clin Inmunol. 2013;132: 1216-19. https://doi.org/10.1016/j.jaci.2013.07.018

3. Branum AM, Lukacs SL. Food Allergy among children in the United States. Pediatrics. 2009;124:1549-55. https://doi.org/ 10.1542/peds.2009-1210

4. Gupta RS, Dyer AA, Jain N, Greenhawt MJ. Childhood food allergies: current diagnosis, treatment, and management strategies. Mayo Clin Proc. 2013;88:512-26. https://doi.org/10.1016/ j.mayocp.2013.03.005

5. Ojeda P, Sastre J, Olaguibel JM, Chivato T. Alergológica 2015: a national survey on allergic diseases in the adult Spanish population. J Investig Allergol Clin Immunol. 2018;28:151-64. https://doi.org/10.18176/jiaci.0264

6. Sicherer SH, Sampson HA. Food allergy. J Allergy Clin Immunol. 2010;125:116-25. https://doi.org/10.1016/j.jaci.2009.08.028
7. Shah E, Pongracic J. Food-induced anaphylaxis: who, what, why and where? Pediatr Ann. 2008;37:536-41. https://doi.org/ 10.3928/00904481-20080801-06

8. Martorell A, Alonso E, Boné J, Echeverría L, López MC, Martín F, et al. Food allergy committee of SEICAP. Position document: IgE-mediated allergy to egg protein. Allergol Immunopathol. 2013;41:320-36. https://doi.org/10.1016/j.aller.2013.03.005

9. Nagakura K, Sato S, Yanagida N, Ebisawa M. Novel immunotherapy and treatment modality for severe food allergies. Curr Opin Allergy Cin Immunol. 2017;17:212-19. https://doi. org/10.1097/ACl.0000000000000365

10. Sampson HA, van Wijk RG, Bindslev-Jensen C, Sicherer SH, Teuber S, Burks W, et al. Standardizing double-blind, placebo-controlled oral food challenges: American Academy of Allergy, Asthma \& Immunology-European Academy of Allergy and Clinical Immunology PRACTALL consensus report. J Allergy Clin Immunol. 2012;130(6):1260-74. https://doi. org/10.1016/j.jaci.2012.10.017

11. Sampson HA, Leung DY, Burks AW, Lack G, Bahna SL, Jones $S M$, et al. A phase II, randomized, double-blind, parallel-group, placebo-controlled oral food challenge trial of Xolair (omalizumab) in peanut allergy. J Allergy Clin Immunol. 2011;127:1309-10. https://doi.org/10.1016/j.jaci.2011.01.051

12. Wood RA, Kim JS, Lindblad R, Nadeau K, Henning AK, Dawson P, et al. A randomized, double-blind, placebo-controlled study of omalizumab combined with oral immunotherapy for the treatment of cow's milk allergy. J Allergy Clin Immunol. 2016;137:1103-10. https://doi.org/10.1016/j.jaci.2015.10.005

13. Bégin $P$, Dominguez $T$, Wilson SP, Bacal L, Mehrotra A, Kausch $B$, et al. Phase 1 results of safety and tolerability in a rush oral immunotherapy protocol to multiple foods using Omalizumab. Allergy, Asthma Clin Immunol. 2014;10:7. https:// doi.org/10.1186/1710-1492-10-7

14. Labrosse R, Graham F, Des Roches A, Bégin P. The use of Omalizumab in food oral immunotherapy. Arch Immunol Therap Exp. 2017;65:189-99. https://doi.org/10.1007/s00005016-0420-z

15. Fiocchi A, Artesani MC, Riccardi C, Mennini M, Pecora V, Fierro $\mathrm{V}$, et al. Impact of omalizumab on food allergy in patients treated for asthma: a real-life study. J Allergy Clin Immunol Pract. 2019;7:1901-09. https://doi.org/10.1016/j. jaip.2019.01.023

16. Mendonca V, Pauperio S, Trindade E, Amil J, Bonito Vitor, A. Omalizumab in pediatric patients with severe food allergy. Allergy. 2011;66:1-104. Abstract 135. https://doi.org/10.1111/ j.1398-9995.2011.02604.x

17. Slavin RG, Ferioli C, Tannenbaum SJ, Martin C, Blogg M, Lowe PJ. Asthma symptom re-emergence after omalizumab withdrawal correlates well with increasing IgE and decreasing pharmacokinetic concentrations. J Allergy Clin Immunol. 2009;123:107-13. https://doi.org/10.1016/j.jaci.2008.09.050 